\title{
MicroRNA-744-5p is downregulated in colorectal cancer and targets SEPT2 to suppress the malignant phenotype
}

\author{
WEI ZHANG ${ }^{1}$, KAI LIAO ${ }^{1}$ and DONGNING LIU ${ }^{2}$ \\ ${ }^{1}$ Department of General Surgery, People's Hospital of Yichun City, Yichun, Jiangxi 336000; \\ ${ }^{2}$ Department of General Surgery, The First Affiliated Hospital of Nanchang University, Nanchang, Jiangxi 330006, P.R. China
}

Received March 13, 2020; Accepted August 28, 2020

DOI: $10.3892 / \mathrm{mmr} .2020 .11692$

\begin{abstract}
MicroRNA (miR)-744-5p serves a pivotal role in the progression of multiple cancers; however, the function of miR-744-5p in colorectal cancer (CRC) remains largely unknown. In the present study, the effects of miR-744-5p on the progression of CRC were analyzed and the mechanisms involved were investigated. It was revealed that miR-744-5p was frequently downregulated in CRC tissues and cell lines. Overexpression of miR-744-5p significantly inhibited the proliferation, colony formation, and promoted the apoptosis of CRC cells. Bioinformatics analysis revealed that Septin 2 (SEPT2) was a potential target of miR-744-5p. miR-744-5p bound the 3'-untranslated region (UTR) of SEPT2 and reduced the level of SEPT2 in CRC cells. A negative correlation between the expression of miR-744-5p and SEPT2 was observed in CRC tissues. Overexpression of SEPT2 counteracted the suppressive effect of miR-744-5p on the proliferation and apoptosis of CRC cells. Collectively, these data demonstrated the functional mechanism of miR-744-5p by targeting SEPT2, which suggested miR-744-5p as a potential target for the treatment of patients with CRC.
\end{abstract}

\section{Introduction}

Colorectal cancer (CRC) is one of the most common malignant cancers $(1,2)$. Although significant improvements have been made in the diagnosis and treatment of CRC, the 5-year survival rate of patients with CRC remains low (3). Numerous patients are diagnosed at an advanced stage, at which point current therapeutic strategies are less efficient due to invasion and metastasis (2). Therefore, it is important to increase understanding of the underlying mechanisms that may contribute to the development of CRC.

Correspondence to: Dr Dongning Liu, Department of General Surgery, The First Affiliated Hospital of Nanchang University, 17 Yongwaizheng Street, Nanchang, Jiangxi 330006, P.R. China E-mail: quedongd22525@126.com

Key words: colorectal cancer, microRNA-744-5p, Septin 2
MicroRNAs (miRNA/miRs) are a class of small, single-stranded RNA with a length of 22 nucleotides and lack protein-coding ability $(4,5)$. miRNAs regulate gene expression at a post-translational level by binding to the 3'-untranslated region (UTR) of target mRNAs, leading to mRNA degradation or translation inhibition $(6,7)$. Due to the widespread function of miRNAs in protein-coding genes, miRNAs play critical roles in multiple cellular processes, including cell proliferation, differentiation and migration (8). Notably, growing evidence has revealed that miRNAs are dysregulated in human cancers and modulate tumor development such as cancer cell metastasis and chemo- or radiotherapeutic sensitivity (9-13). Aberrant expression of miRNA has previously been revealed in CRC and possibly serves as a potential target to disrupt CRC progression (14-16). For example, a decreased serum level of miR-98 predicted an unfavorable clinical outcome of patients with CRC (17). A recent study also demonstrated that miR-361 promoted the sensitivity of CRC cells to 5-fluorouracil, indicating miR-361 as a possible drug target to suppress tumorigenesis (18). Notably, recent studies revealed the significantly dysregulated expression of miR-744-5p in multiple cancers, such as hepatocellular carcinoma, gastric cancer and pancreatic cancer (19-21). Dysfunction of miR-744-5p has been suggested to play suppressive roles in the development of cancers. However, its role in CRC and the underlying mechanisms are unknown.

Septin 2 (SEPT2) is a novel GTP-binding cytoskeletal protein that is evolutionarily conserved and structurally associated with the RAS oncogene (22). Numerous studies have indicated the link between the altered expression of SEPT2 in tumorigenesis (23-25). Downregulation of SEPT2 suppressed the growth of hepatocellular carcinoma cells and induced cell cycle arrest in glioma, and led to reduced cell viability (26). Overexpression of SEPT2 results in cytokinesis failure, centrosome amplification, and the formation of multipolar spindle bodies that frequently occur in cancer cells (27). Although the function of SEPT2 has been demonstrated in cancers, the involvement of SEPT2 in CRC warrants further study.

The present study investigated the expression pattern of miR-744-5p in CRC tissues and characterized the functional mechanisms. It was revealed that miR-744-5p was downregulated in CRC tissues and cell lines. Consequently, overexpression of miR-744-5p inhibited the growth of CRC cells via targeting SEPT2. The findings presented reveal a 
novel role of miR-744-5p/SEPT2 signaling in the progression of CRC.

\section{Materials and methods}

Tissue samples. The paired CRC tissues and matched adjacent normal tissues were obtained from 50 patients (age, 36-75 years; 22 women and 28 men) who underwent surgery at the People's Hospital of Yichun City between May 2011 and September 2013. Patients who were treated with chemotherapy or radiotherapy were excluded from this study. Tissues were frozen in the liquid immediately and stored at $-80^{\circ} \mathrm{C}$ until use. Written informed consent was obtained from all the patients. The usage of the tissues was approved by the Ethics Committee of the People's Hospital of Yichun City (approval no. 2014041566) on April 15, 2014.

Cell culture and transfection. Human CRC cell lines HCT116, SW480, SW620, RKO and normal colon cell line CCD-18Co were obtained from the Cell Bank of the Chinese Academy of Sciences. Cells were grown in DMEM (Thermo Fisher Scientific, Inc.) containing 10\% FBS (Thermo Fisher Scientific, Inc.) at $37^{\circ} \mathrm{C}$ in a humidified atmosphere containing $5 \% \mathrm{CO}_{2}$.

The miR-744-5p mimics (5'-UGCGGGGCUAGGGCUA ACAGCA-3') and miRNA negative control (miR-NC; 5'-GAG CUACGGUAGAGCCGGUAGC-3') were obtained from Guangzhou RiboBio Co., Ltd. A total of $50 \mathrm{nM}$ miRNA was transfected into cells using the Lipofectamine ${ }^{\circledR} 2000$ reagent (Invitrogen; Thermo Fisher Scientific, Inc.) according to the manufacturer's instructions. After transfection for $48 \mathrm{~h}$, cells were harvested for subsequent experiments.

Reverse transcription-quantitative PCR (RT-qPCR). Total RNA was isolated from tissues and cells using TRIzol ${ }^{\circledR}$ reagent.(Beyotime Institute of Biotechnology) cDNA was synthesized with the Maxima First Strand cDNA Synthesis kit (Thermo Fisher Scientific, Inc.) according to the manufacturer's protocol. The expression level of miR-744-5p was determined using the AceQ qPCR SYBRGreen Master Mix (Vazyme Biotech Co.) on the CFX Connect Real-Time System (Bio-Rad Laboratories Inc). The expression of U6 was also detected for normalization. The specific primer sequences were as follows: miR-744-5p forward, 5'-AATGCGGGGCT AGGGCTA-3' and reverse, 5'-GTGCAGGGTCCGAGGT-3'; U6 forward, 5'-CTCGCTTCGGCAGCACA-3' and reverse, 5'-AACGCTTCACGAATTTGCGT-3'; SEPT2 forward, 5'-TA AACAGCCTATTCCTAACT-3' and reverse, 5'-CATAAACG CCACATCTAA-3'; HNRNPC forward, 5'-AGAACCCGGG AGTAGGAGAC-3' and reverse, 5'-TCTCACAAAGCCGA AAACAA-3'; NFIX forward, 5'-ACTCCCCGTACTGC CTCAC-3' and reverse, 5'-TGCAGGTTGAACCAGGTGTA-3'; c-Myc forward, 5'-CGTCCTCGGATTCTCTGCTC-3' and reverse, 5'-GCTGGTGCATTTTCGGTTCT-3'; PAX2 forward, 5'-CCTCGCTCCAATGGTGAGAA-3' and reverse, 5'-TGCTGCTGGGTGAAGGTGTC-3'; GAPDH forward, 5'-ACACCCACTCCTCCACCTTT-3' and reverse, 5'-TTA CTCCTTGGAGGCCATGT-3'. The PCR conditions were set as: Denaturation at $95^{\circ} \mathrm{C}$ for $5 \mathrm{~min}$, followed by 40 cycles of denaturation at $95^{\circ} \mathrm{C}$ for $15 \mathrm{sec}$, and annealing and extension at $60^{\circ} \mathrm{C}$ for $45 \mathrm{sec}$. The relative gene expression of miR-744-5p was analyzed using the $2^{-\Delta \Delta \mathrm{Cq}}$ method (28).

Cell proliferation assay. The proliferation of CRC cells transfected with miR-744-5p mimics or miRNA negative control was determined by the Cell Counting Kit-8 (CCK- 8 , cat. no. C0038; Beyotime Institute of Biotechnology) assay according to the manufacturer's instructions. CRC cells were seeded in a 96-well plate at the density of 1,000 cells/ well and cultured overnight. Then, $10 \mu \mathrm{l}$ CCK- 8 reagent was added into the medium after $24 \mathrm{~h}$ and incubated for an additional $4 \mathrm{~h}$ at $37^{\circ} \mathrm{C}$. The absorbance at $450 \mathrm{~nm}$ for each well was detected by an Epoch microplate (Bio-Tek Instruments). The assay was performed in triplicate.

Colony formation assay. The CRC cells transfected with the miR-744-5p mimics or miRNA negative control were seeded into the 6 -well plate with 600 cells/well. Cells were grown with DMEM containing 10\% FBS and cultured for 10 days. The colonies were stained with $0.5 \%$ crystal violet (Beyotime Institute of Biotechnology) room temperature (RT) for $10 \mathrm{~min}$ after fixation with $70 \%$ ethanol (Solarbio) at RT for $10 \mathrm{~min}$. The colonies were counted using a light microscope (magnification, $\mathrm{x} 50$ ).

Dual-luciferase reporter assay. The wild-type (WT) or mutant (MT) 3'-UTR sequences of SEPT2 containing miR-744-5p binding sites were amplified and inserted into the psiCHECK2 vector (Promega Corporation). CRC cells were transfected with miR-744-5p mimics or miR-NC with psiCHECK2-WT-SEPT2-3'UTR or psiCHECK2-MT-SEPT2-3'UTR. After transfection with Lipofectamine ${ }^{\circledR}$ for $48 \mathrm{~h}$, cells were harvested and the luciferase activity was determined using the Dual-Luciferase Reporter Assay (Promega Corporation) according to the manufacturer's protocol. The activity of Renilla luciferase was also detected for normalization.

Western blotting. Total proteins were extracted from CRC cells using an NP-40 lysis buffer (Beyotime Institute of Biotechnology) and the protein concentration was determined using a Bicinchoninic Acid Protein Assay kit (Beyotime Institute of Biotechnology). A total of $20 \mu \mathrm{g}$ proteins were separated by $15 \%$ SDS-PAGE and transferred onto the nitrocellulose membranes (EMD Millipore). After blocking with $5 \%$ non-fat milk for $1 \mathrm{~h}$ at room temperature (RT), the membranes were probed with primary antibodies against SEPT2 (1:2,000; cat. no. 11397-1-AP; Thermo Fisher Scientific, Inc.) or GAPDH (1:3,000; cat. no. ab9485; Abcam) at $4^{\circ} \mathrm{C}$ overnight. Subsequently, membranes were incubated with HRP-conjugated goat anti-Rabbit IgG H\&L secondary antibody $(1: 3,000$; cat. no. ab205718, Abcam) at RT for $1 \mathrm{~h}$. The blots were developed by an Enhanced Chemiluminescence (ECL) system (Thermo Fisher Scientific, Inc.). The antibodies used in this study, including anti-SEPT2 and anti-GAPDH (both from ProteinTech Group, Inc.) were commercially obtained.

Cell apoptosis. The cell apoptosis was detected using the FITC Annexin V Apoptosis Detection kit (Beijing Solarbio Science \& Technology Co., Ltd.) according to the manufac- 

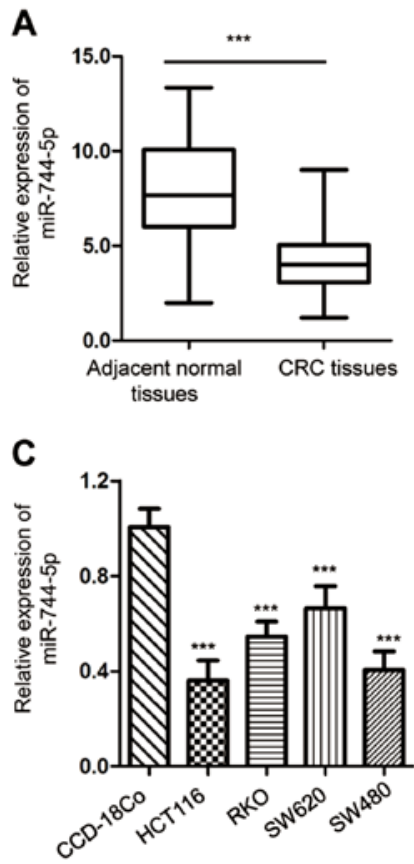

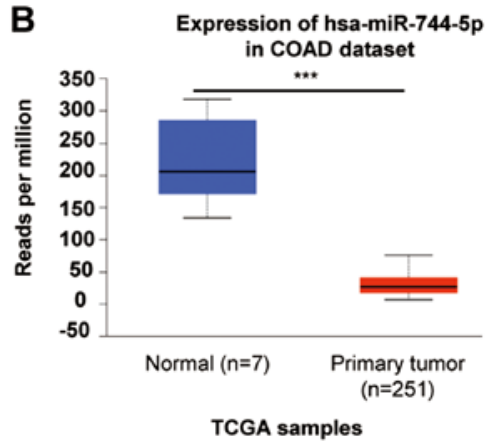

Figure 1. miR-744-5p is downregulated in CRC. (A) The expression of miR-744-5p in paired CRC tissues and adjacent normal tissues was detected by RT-qPCR. (B) The expression of miR-744-5p in CRC tissues and normal tissues was predicted using the TCGA-COAD database. (C) The level of miR-744-5p in CRC cell lines and normal CCD-18Co cells was compared using RT-qPCR analysis. ${ }^{* * *} \mathrm{P}<0.001$. CRC, colorectal cancer; miR, microRNA, RT-qPCR, reverse transcription-quantitative PCR.

turer's instructions. Briefly, $5 \times 10^{5} \mathrm{CRC}$ cells were transfected and harvested after $48 \mathrm{~h}$. Cells were washed with pre-cold PBS and re-suspended with the provided binding buffer. Cells were then incubated with Annexin V-FITC for $15 \mathrm{~min}$ at RT in the dark and subsequently stained with PI for at RT for 1 min. The cell apoptosis was determined with the FACScan flow cytometer (BD Biosciences). The data were analyzed using FlowJo software (v10.7, https://www.flowjo.com/solutions/flowjo/downloads).

Targets prediction. The possible targets of miR-744-5p were predicted with the miRDB (http://mirdb.org/; version 6.0) online dataset.

Bioinformatics. Gene expression in CRC tissues and normal tissues was evaluated against The Cancer Genome Atlas Colon Adenocarcinoma (TCGA-COAD) database (http://ualcan. path.uab.edu/index.html) (29).

Statistical analysis. Data were presented as the mean \pm standard deviation and analyzed with SPSS 22.0 (IBM Corp.). Differences between groups were determined with unpaired Student's t-test or one-way ANOVA followed by Tukey's post hoc tests. The difference shown in Figs. 1A and 4A was analyzed using paired Student's t-test. The correlation between miR-744-5p and SEPT2 was analyzed by a Spearman's correlation test. $\mathrm{P}<0.05$ was considered to indicate a statistically significant difference.

\section{Results}

miR-744-5p is downregulated in CRC. To explore the potential involvement of miR-744-5p in CRC, the expression pattern of miR-744-5p in CRC tissues and paired adjacent normal tissues was detected by RT-qPCR. The results revealed that the expression level of miR-744-5p was significantly reduced in CRC tissues compared with the surrounding non-cancerous tissues (Fig. 1A). To support this result, the expression of miR-744-5p in CRC tissues and normal tissues was also evaluated against the TCGA-COAD database (http://ualcan.path.uab.edu/index.html) (29). Consistent with the presented findings, there was a significantly reduced level of miR-744-5p observed in CRC tissues compared with the normal tissues (Fig. 1B). Additionally, miR-744-5p expression in CRC cells and normal cells was analyzed. It was determined that miR-744-5p was significantly decreased in CRC cell lines compared with that in the normal cell line CCD-18Co (Fig. 1C). These results indicated the downregulation of miR-744-5p in CRC.

miR-744-5p inhibits the proliferation and induces apoptosis in CRC cells. To investigate the function of miR-744-5p in CRC, both HCT116 and SW480 cells were selected for biological analysis due to the relatively low miR-744-5p in these cells. The miR-744-5p mimic oligonucleotides were transfected into CRC cells and the expression of miR-744-5p was detected by RT-qPCR (Fig. 2A). The CCK-8 assay revealed that miR-744-5p overexpression significantly inhibited the proliferation of both HCT116 and SW480 cells compared with cells transfected with miR-NC (Fig. 2B and C). A colony formation assay was performed to confirm the suppressive role of miR-744-5p in $\mathrm{CRC}$. The results indicated that overexpression of miR-744-5p inhibited the colony formation ability of both HCT116 and SW480 cells (Fig. 2D). Consistent with these results, highly expressed miR-744-5p also significantly upregulated the apoptosis of CRC cells (Fig. 2E). These findings indicated 

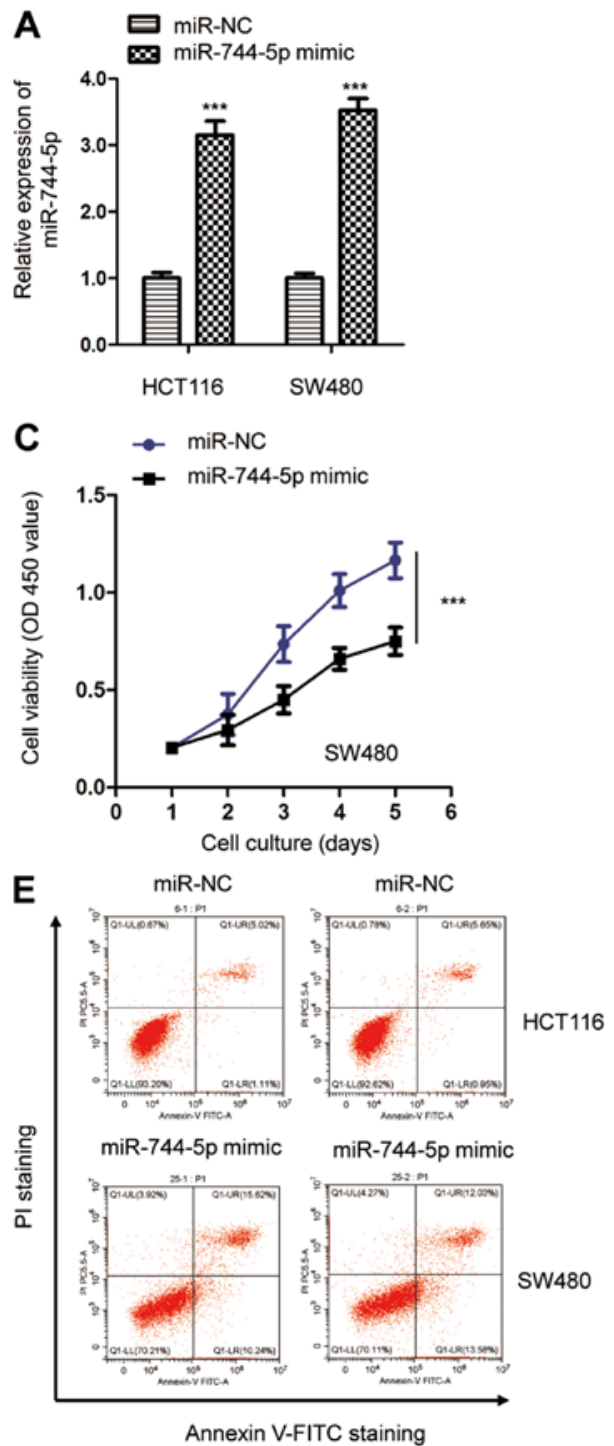
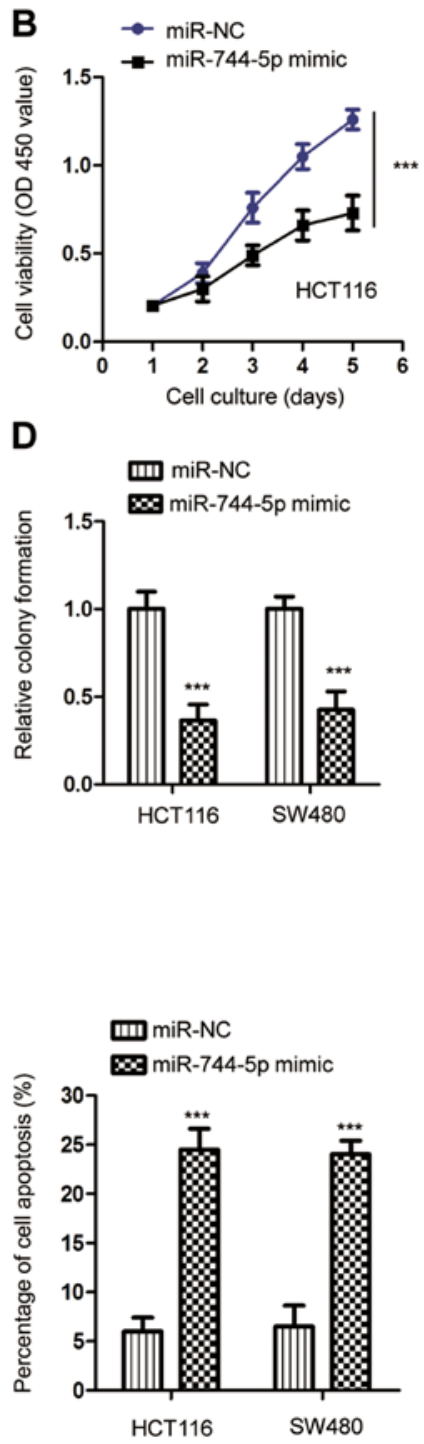

Figure 2. Overexpression of miR-744-5p inhibits the proliferation of CRC cells. (A) The relative expression of miR-744-5p in CRC cells transfected with the miR-744-5p mimic or miR-NC. (B and C) A CCK-8 assay was performed to determine the proliferation of CRC cells expressing the miR-744-5p mimic or miR-NC. (D) Overexpression of miR-744-5p significantly reduced the colony formation of both HCT116 and SW480 cells. (E) Transfection of miR-744-5p increased the apoptosis of CRC cells compared with cells expressing miR-NC. ${ }^{* * *} \mathrm{P}<0.001$. CRC, colorectal cancer; miR, microRNA; miR-NC, microRNA control; CCK-8, Cell Counting Kit-8.

the potential tumor-suppressive function of miR-744-5p in the development of CRC.

SEPT2 is a target of miR-744-5p in CRC. To understand the molecular mechanism underlying the tumor-suppressive role of miR-744-5p in CRC, the miRDB database was used to predict the potential binding targets of miR-744-5p (29). It was determined that the 3'-UTR of SEPT2 contains a putative binding site for miR-744-5p (Fig. 3A). To confirm this predicted binding, a dual-luciferase assay was performed by transfecting miR-744-5p mimics and the luciferase reporter vector carrying WT or MT 3'-UTR of SEPT2. The data revealed that overexpression of miR-744-5p significantly decreased the luciferase activity of WT but not MT 3'-UTR of SEPT2 (Fig. 3B and C). To detect whether miR-744-5p regulates the expression of SEPT2, RT-qPCR and western blot assays were performed to examine the mRNA and protein levels of SEPT2, respectively. The results revealed that overexpression of miR-744-5p significantly reduced the mRNA level of SEPT2 in CRC cells (Fig. 3D). Similarly, the protein expression of SEPT2 was also suppressed by the transfection of miR-744-5p in both HCT116 and SW480 cells (Fig. 3E). These results demonstrated that miR-744-5p targeted SEPT2 and negatively modulated the expression of SEPT2 in CRC.

SEPT2 is upregulated in CRC and is inversely correlated with the expression of miR-744-5p. To further explore the relationship between the level of SEPT2 and miR-744-5p, the expression of SEPT2 in CRC tissues and paired adjacent normal tissues was detected. The RT-qPCR data revealed that SEPT2 expression was significantly increased in CRC tissues compared with the surrounding non-cancerous tissues (Fig. 4A). The level of SEPT2 was also higher in CRC cells than in normal CCD-18Co cells (Fig. 4B). Additionally, the correlation between the expression of miR-744-5p and SEPT2 was analyzed using Spearman's correlation test. The data 


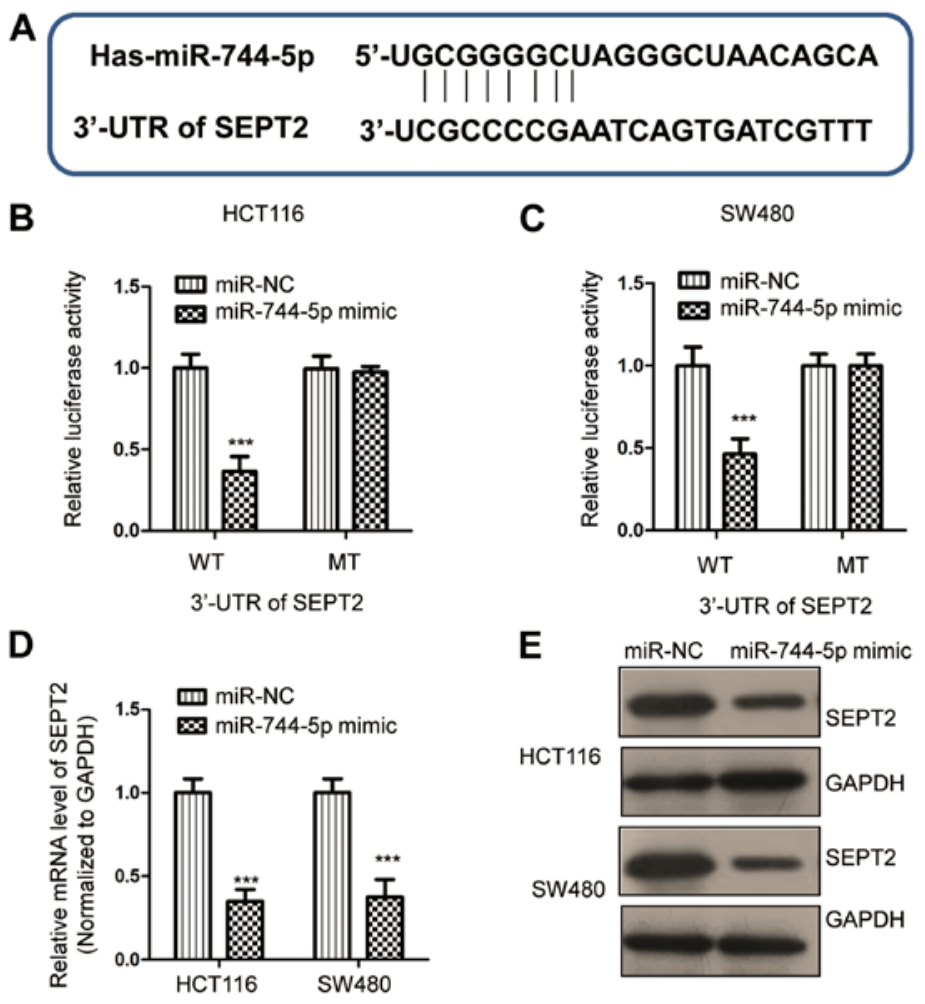

Figure 3. SEPT2 is a target of miR-744-5p. (A) The predicted binding site of miR-744-5p at the 3'-UTR of SEPT2. (B and C) Luciferase activity of CRC cells expressing miR-744-5p and WT or MT 3'-UTR of SEPT2. (D) The mRNA level of SEPT2 in CRC cells transfected with the miR-744-5p mimic or miR-NC. (E) Overexpression of miR-744-5p decreased the protein level of SEPT2 in both HCT116 and SW480 cells. ${ }^{* * *} \mathrm{P}<0.001$. CRC, colorectal cancer; miR, microRNA; miR-NC, micRNA control; SEPT2, septin 2; WT, wild-type; MT, mutant.

A

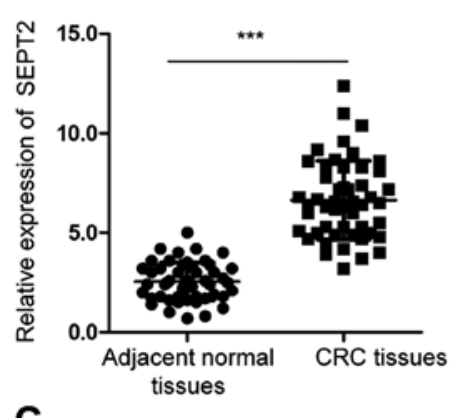

C

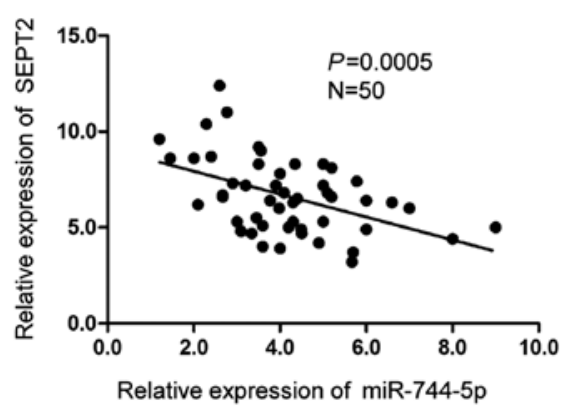

B

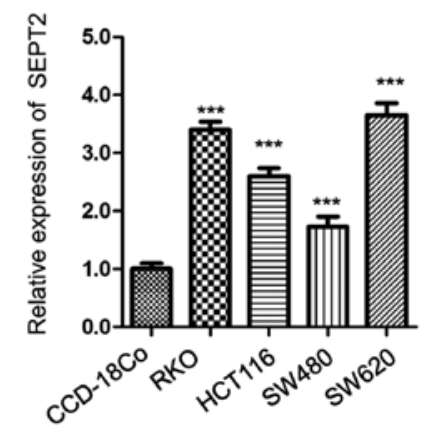

Figure 4. SEPT2 is highly expressed in CRC tissues. (A) The mRNA levels of SEPT2 in CRC tissues and paired adjacent normal tissues was detected by RT-qPCR analysis. (B) The expression of SEPT2 in CRC cell lines and normal CCD-18Co cells was compared by RT-qPCR. (C) The correlation between the expression of SEPT2 and miR-744-5p in CRC tissues was determined by the Spearman's correlation test. ${ }^{* * *} \mathrm{P}<0.001$. CRC, colorectal cancer; miR, microRNA, RT-qPCR, reverse transcription-quantitative PCR; SEPT2, Septin 2.

revealed that the expression of SEPT2 was inversely correlated with the level of miR-744-5p in CRC tissues (Fig. 4C). The findings of the present study supported the conclusion that SEPT2 was a target of miR-744-5p in CRC. 

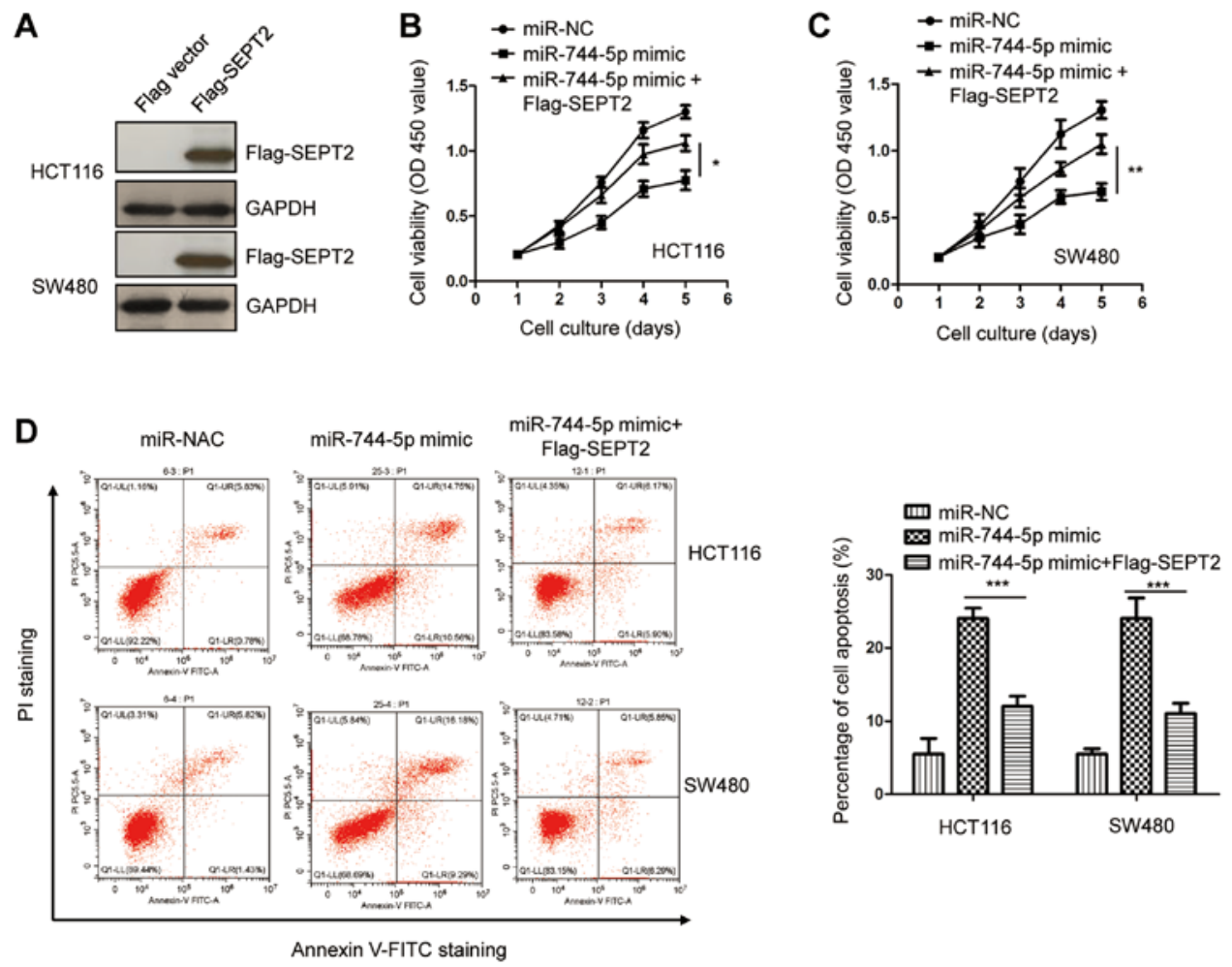

Figure 5. Restoration of SEPT2 attenuates the suppressive role of miR-744-5p in CRC. (A) Transfection of Flag-SEPT2 was confirmed by western blotting against an anti-Flag antibody. (B and C) Overexpression of SEPT2 significantly attenuated the inhibitory effects of miR-744-5p in regulating the proliferation of CRC cells. (D) Reintroduction of SEPT2 reversed miR-744-5p-induced apoptosis of CRC cells. ${ }^{*} \mathrm{P}<0.05 ;{ }^{* *} \mathrm{P}<0.01 ;{ }^{* * *} \mathrm{P}<0.001$. CRC, colorectal cancer; miR, microRNA; SEPT2, Septin 2.

Overexpression of SEPT2 reverses the tumor-suppressive effects of miR-744-5p in CRC. To investigate whether SEPT2 plays a suppressive role for miR-744-5p in the malignancy of CRC, SEPT2 was overexpressed by transfecting pcDNA-3Flag-SEPT2 into both HCT116 and SW480 cells. The ectopic expression of SEPT2 was validated by western blotting (Fig. 5A). The results of the CCK-8 assay revealed that SEPT2 overexpression reversed the suppressive effects of miR-744-5p mimics on CRC cell proliferation (Fig. 5B and C). Additionally, the apoptosis of CRC cells was significantly decreased with the co-transfection of SEPT2 compared with cells only expressing miR-744-5p mimics (Fig. 5D). These results indicated that miR-744-5p partially inhibited the progression of CRC by downregulating SEPT2. Notably, recent studies reported that miR-744-5p targets c-Myc in papillary thyroid carcinoma cells (30), Heterogeneous Nuclear Ribonucleoprotein C (HNRNPC) and Nuclear Factor I X (NFIX) in ovarian cancer cells (19), and Paired box gene 2 (PAX2) in non-small cell lung cancer (NSCLC) (20). To determine the correlation between miR-744-5p and the aforementioned gene expression, the expression change of HNRNPC, NFIX, c-Myc and PAX2 in CRC tissues and normal tissues was examined via TCGA-COAD database. No significant change for the expression of NFIX and PAX2 were revealed in CRC tissues compared with that of the normal tissues (Fig. 6A and B). It was also revealed that the expression of NFIX and PAX2 was not significantly reduced by the transfection of miR-744-5p in HCT116 and SW480 cells (Fig. 6C and D). In contrast, highly expressed HNRNPC and c-Myc was revealed in CRC tissues compared with the normal tissues by the TCGA-COAD
(Fig. 6E and F). Decreased levels of HNRNPC and c-Myc were observed with the transfection of miR-744-5p in HCT116 and SW480 cells by RT-qPCR (Fig. 6G and H). Additionally, the expression of HNRNPC and c-Myc in CRC tissues was analyzed for their correlation with miR-744-5p. An inverse correlation was observed between the levels of HNRNPC, c-Myc and miR-744-5p, respectively (Fig. 6I and J). These results indicated the possibility of HNRNPC and c-Myc as potential targets of miR-744-5p in CRC in addition to SEPT2.

\section{Discussion}

$\mathrm{CRC}$ is one of the most common malignant tumors worldwide and a major cause of cancer-related mortality (31). Growing evidence suggests that miRNA acts as a tumor suppressor or promoter in the progression and metastasis of CRC by regulating cell proliferation, apoptosis, and cell cycles (14). The present study provided novel insights into the tumor inhibitory effects of miR-744-5p in the tumorigenesis of CRC. The present study detected the expression level of miR-744-5p in CRC tissues and cell lines. The function and underlying mechanisms of miR-744-5p that are responsible for the effects of miR-744-5p in the development of CRC were also investigated.

miR-744-5p has previously been revealed to be significantly downregulated in cancers $(19,20,32)$. Overexpression of miR-744-5p induced apoptosis of ovarian cancer cells and high miR-744-5p expression was associated with prolonged disease-free survival of patients with ovarian cancer (19). Additionally, the serum level of miR-744-5p was dysregulated 

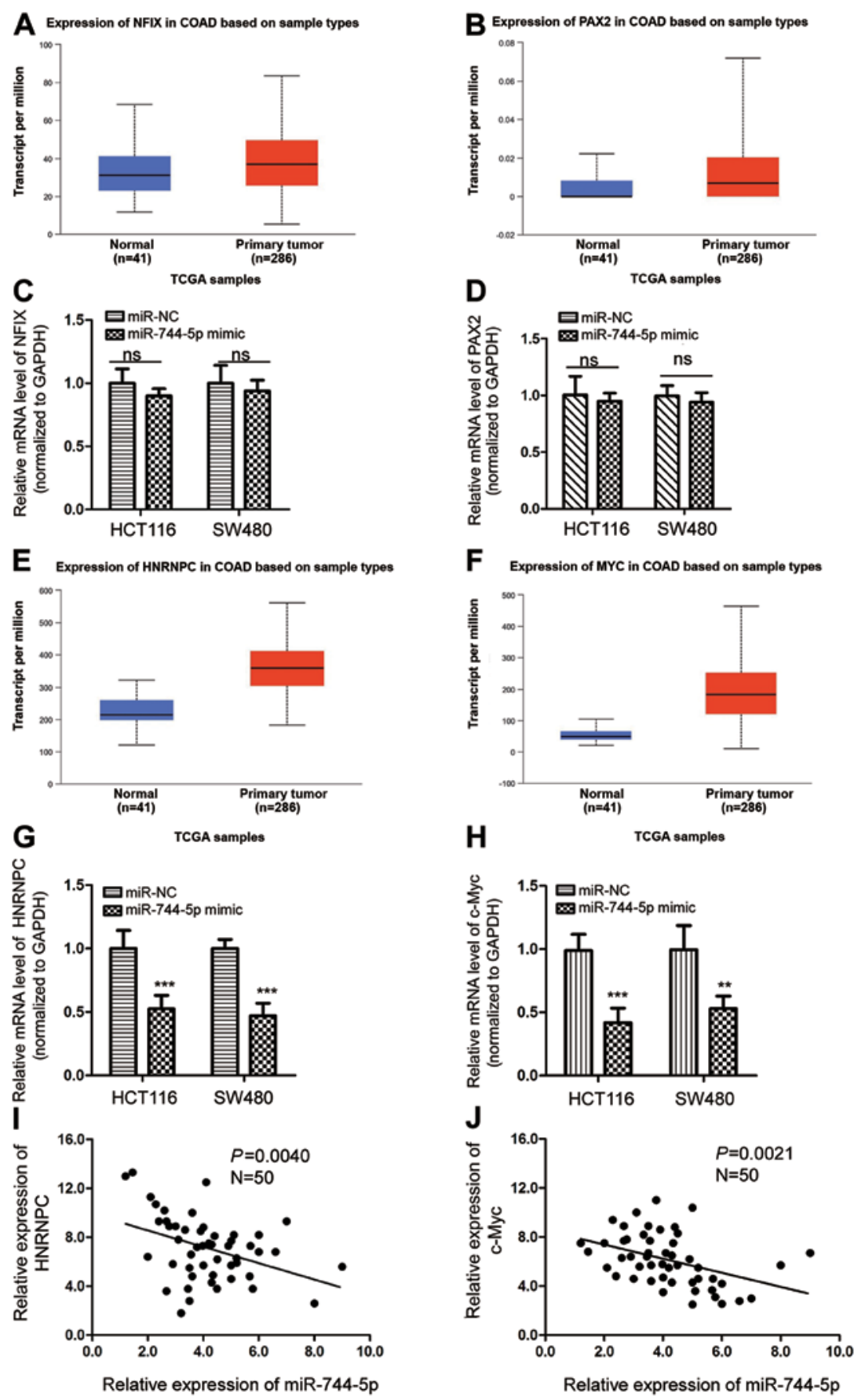

Figure 6. Correlation between the levels of miR-744-5p with other possible targets. (A and B) The expression of NFIX and PAX2 in CRC tissues and normal tissues was evaluated with the TCGA-COAD database. (C and D) HCT116 and SW480 cells were transfected with miR-NC or miR-744-5p mimics, and the mRNA abundance of NFIX and PAX2 was detected by RT-qPCR. (E and F) The expression of HNRNPC and c-Myc in CRC tissues and normal tissues was evaluated with the TCGA-COAD database. ( $\mathrm{G}$ and $\mathrm{H}$ ) The mRNA levels of HNRNPC and c-Myc were examined by RT-qPCR with CRC cells expressing miR-NC or miR-744-5p mimics. (I and J) The correlation between the expression of miR-744-5p and HNRNPC, c-Myc in CRC tissues was determined by the Spearman's correlation test, respectively. ${ }^{* *} \mathrm{P}<0.01 ;{ }^{* * *} \mathrm{P}<0.001$. CRC, colorectal cancer; miR, microRNA; RT-qPCR, reverse transcription-quantitative PCR; NFIX, Nuclear Factor I X; PAX2, Paired box gene 2; HNRNPC, Heterogeneous Nuclear Ribonucleoprotein C; miR-NC, micRNA control; Ns, no significance.

in various types of cancers, such as pancreatic and biliary tract cancers, which indicates the potential significance of miR-744-5p in the diagnosis of cancers (21). A recent study also reported decreased expression of miR-744-5p in NSCLC (20). Overexpression of miR-744-5p inhibited the proliferation, colony formation, and invasion of NSCLC cells. In the present study, miR-744-5p was significantly downregulated in CRC tissues and cell lines. Transfection of miR-744-5p suppressed proliferation and colony formation, and induced apoptosis of
CRC cells. These observations suggested that miR-744-5p may be a potential therapeutic target for the treatment of patients with CRC.

To investigate the molecular mechanism underlying the tumor-suppressive effects of miR-744-5p in CRC, the potential targets of miR-744-5p were predicted with bioinformatics software. The 3'-UTR of SEPT2 was found to carry the binding sequence for miR-744-5p. SEPT2 is a conserved filamentous GTPase and is overexpressed in a variety of 
human cancers (33). SEPT2 acts as a cancer-promoting gene and is associated with the poor prognosis of patients with cancer (34). However, the regulatory mechanism of SEPT2 in cancer remains unclear. In the present study, miR-744-5p bound the 3'-UTR of SEPT2 and inhibited the expression of SEPT2 in CRC cells. The level of SEPT2 was increased in CRC tissues compared with the matched normal tissues. The upregulation of SEPT2 was significantly inversely correlated with the level of miR-744-5p in CRC tissues. The reintroduction of exogenously expressed SEPT2 abrogated the suppressive function of miR-744-5p in the proliferation of CRC cells. The present results demonstrated that miR-744-5p targeted SEPT2 and inhibited the malignant phenotype of CRC cells.

In conclusion, the present study indicated that miR-744-5p was downregulated in CRC. Overexpression of miR-744-5p significantly restricted the proliferation and promoted apoptosis of CRC cells by targeting SEPT2. Hence, the miR-744-5p/SEPT2 axis may represent a potential therapeutic target for patients with CRC. The limitation of this study was that most of the conclusions were obtained from in vitro assays. Therefore, further investigation is required to examine the critical function of miR-744-5p/SEPT2 signaling in the progression of CRC using an in vivo study. Another limitation is that only 50 patients were enrolled in the present study to evaluate the clinical significance of miR-744-5p in CRC. A larger sample size is required to investigate the association between miR-744-5p expression and the prognosis of patients with CRC.

\section{Acknowledgements}

Not applicable.

\section{Funding}

No funding was received.

\section{Availability of data and materials}

The datasets used and/or analyzed during the current study are available from the corresponding author on reasonable request.

\section{Authors' contributions}

All authors (WZ, KL and DL) contributed to the project design, data acquisition and analysis, manuscript writing and gave final approval of the version to be published. All authors have read and approved the final manuscript.

\section{Ethics approval and consent to participate}

The usage of patient tissues was approved by the Ethics Committee of the People's Hospital of Yichun City (approval no. 2014041566) on April 15, 2014. Written informed consent was obtained from all the patients.

\section{Patient consent for publication}

Not applicable.

\section{Competing interests}

The authors declare that they have no competing interests.

\section{References}

1. Weinberg BA, Marshall JL and Salem ME: The Growing Challenge of Young Adults With Colorectal Cancer. Oncology (Williston Park) 31: 381-389, 2017.

2. Yiu AJ and Yiu CY: Biomarkers in Colorectal Cancer. Anticancer Res 36: 1093-1102, 2016.

3. Kuipers EJ, Grady WM, Lieberman D, Seufferlein T, Sung JJ, Boelens PG, van de Velde CJ and Watanabe T: Colorectal cancer. Nat Rev Dis Primers 1: 15065, 2015.

4. Cai Y, Yu X, Hu S and Yu J: A brief review on the mechanisms of miRNA regulation. Genomics Proteomics Bioinformatics 7: 147-154, 2009.

5. Mohr AM and Mott JL: Overview of microRNA biology. Semin Liver Dis 35: 3-11, 2015.

6. Bartel DP: MicroRNAs: Genomics, biogenesis, mechanism, and function. Cell 116: 281-297, 2004.

7. Fabian MR, Sonenberg N and Filipowicz W: Regulation of mRNA translation and stability by microRNAs. Annu Rev Biochem 79: 351-379, 2010.

8. Krol J, Loedige I and Filipowicz W: The widespread regulation of microRNA biogenesis, function and decay. Nat Rev Genet 11: 597-610, 2010.

9. Kwak PB, Iwasaki S and Tomari Y: The microRNA pathway and cancer. Cancer Sci 101: 2309-2315, 2010.

10. Farazi TA, Spitzer JI, Morozov P and Tuschl T: miRNAs in human cancer. J Pathol 223: 102-115, 2011.

11. Qu H, Xu W, Huang Y and Yang S: Circulating miRNAs: Promising biomarkers of human cancer. Asian Pac J Cancer Prev 12: 1117-1125, 2011.

12. Gentilin E, Degli Uberti E and Zatelli MC: Strategies to use microRNAs as therapeutic targets. Best Pract Res Clin Endocrinol Metab 30: 629-639, 2016.

13. Iorio MV and Croce CM: MicroRNA dysregulation in cancer: Diagnostics, monitoring and therapeutics. A comprehensive review. EMBO Mol Med 9: 852, 2017.

14. Sur DG, Colceriu M, Sur G, Aldea C, Silaghi C, Samasca G, Lupan I, Căinap C, Burz C and Irimie A: MiRNAs roles in the diagnosis, prognosis and treatment of colorectal cancer. Expert Rev Proteomics 16: 851-856, 2019.

15. Wei L, Wang X, Lv L, Zheng Y, Zhang N and Yang M: The emerging role of noncoding RNAs in colorectal cancer chemoresistance. Cell Oncol (Dordr) 42: 757-768, 2019.

16. Wan TMH, Iyer DN and Ng L: Roles of microRNAs as non-invasive biomarker and therapeutic target in colorectal cancer. Histol Histopathol 35: 225-237, 2020.

17. Wang YG, He Q, Guo SQ and Shi ZZ: Reduced serum miR-98 predicts unfavorable clinical outcome of colorectal cancer. Eur Rev Med Pharmacol Sci 23: 8345-8353, 2019.

18. Zhang L, Li B, Zhang B, Zhang H and Suo J: miR-361 enhances sensitivity to 5-fluorouracil by targeting the FOXM1-ABCC5/10 signaling pathway in colorectal cancer. Oncol Lett 18: 4064-4073, 2019.

19. Kleemann M, Schneider H, Unger K, Sander P, Schneider EM, Fischer-Posovszky P, Handrick R and Otte K: miR-744-5p inducing cell death by directly targeting HNRNPC and NFIX in ovarian cancer cells. Sci Rep 8: 9020, 2018.

20. Chen S, Shi F, Zhang W, Zhou Y and Huang J: miR-744-5p inhibits non-small cell lung cancer proliferation and invasion by directly targeting PAX2. Technol Cancer Res Treat 18: $1533033819876913,2019$.

21. Kim K, Yoo D, Lee HS, Lee KJ, Park SB, Kim C, Jo JH, Jung DE and Song SY: Identification of potential biomarkers for diagnosis of pancreatic and biliary tract cancers by sequencing of serum microRNAs. BMC Med Genomics 12: 62, 2019.

22. Dolat L, Hu Q and Spiliotis ET: Septin functions in organ system physiology and pathology. Biol Chem 395: 123-141, 2014.

23. Cerveira N, Correia C, Bizarro S, Pinto C, Lisboa S, Mariz JM, Marques M and Teixeira MR: SEPT2 is a new fusion partner of MLL in acute myeloid leukemia with $\mathrm{t}(2 ; 11)(\mathrm{q} 37 ; \mathrm{q} 23)$. Oncogene 25: 6147-6152, 2006.

24. Angelis D and Spiliotis ET: Septin Mutations in Human Cancers. Front Cell Dev Biol 4: 122, 2016. 
25. Yu W, Ding X, Chen F, Liu M, Shen S, Gu X and Yu L: The phosphorylation of SEPT2 on Ser218 by casein kinase 2 is important to hepatoma carcinoma cell proliferation. Mol Cell Biochem 325: 61-67, 2009.

26. Xu D, Liu A, Wang X, Chen Y, Shen Y, Tan Z and Qiu M: Repression of Septin9 and Septin2 suppresses tumor growth of human glioblastoma cells. Cell Death Dis 9: 514, 2018.

27. Neubauer K and Zieger B: The Mammalian Septin Interactome. Front Cell Dev Biol 5: 3, 2017.

28. Livak KJ and Schmittgen TD: Analysis of relative gene expression data using real-time quantitative PCR and the 2(-Delta Delta C(T)) Method. Methods 25: 402-408, 2001.

29. Chandrashekar DS, Bashel B, Balasubramanya SAH, Creighton CJ, Ponce-Rodriguez I, Chakravarthi BVSK and Varambally S: UALCAN: A portal for facilitating tumor subgroup gene expression and survival analyses. Neoplasia 19: 649-658, 2017.

30. Yuan Q, Fan Y, Liu Z, Wang X, Jia M, Geng Z, Zheng J and Lu X: miR-744-5p mediates lncRNA HOTTIP to regulate the proliferation and apoptosis of papillary thyroid carcinoma cells. Exp Cell Res 392: 112024, 2020.
31. Azeem S, Gillani SW, Siddiqui A, Jandrajupalli SB, Poh V and Syed Sulaiman SA: Diet and colorectal cancer risk in Asia - a systematic review. Asian Pac J Cancer Prev 16: 5389-5396, 2015.

32. Sui $Y$, Lin G, Zheng Y and Huang W: LncRNA MAFG-AS1 boosts the proliferation of lung adenocarcinoma cells via regulating miR-744-5p/MAFG axis. Eur J Pharmacol 859: 172465, 2019.

33. Fung KY, Dai L and Trimble WS: Cell and molecular biology of septins. Int Rev Cell Mol Biol 310: 289-339, 2014.

34. Liu M, Shen S, Chen F, Yu W and Yu L: Linking the septin expression with carcinogenesis. Mol BiolRep 37: 3601-3608, 2010.

This work is licensed under a Creative Commons Attribution-NonCommercial-NoDerivatives 4.0 International (CC BY-NC-ND 4.0) License. 